\title{
Risk Factors, Diagnosis, and Current Practices in the Management of Intraventricular Hemorrhage in Preterm Infants: A Review
}

\author{
Khaled El-Atawi ${ }^{1}$, Mahmoud Elhalik ${ }^{2}$, Tushar Kulkarni ${ }^{3}$, Amany Abdelsamed ${ }^{4}$, Lois Alexander ${ }^{4}$ and \\ Aswathy Devaki Satyan ${ }^{4}$ \\ ${ }^{1}$ Consultant Neonatologist, Neonatal Intensive Care, Pediatric Department, Latifa Hospital, Dubai Health Authority, UAE \\ ${ }^{2}$ Consultant Neonatologist and Head of Pediatric Department, Neonatal Intensive Care, Pediatric Department, Latifa Hospital, Dubai Health \\ Authority, UAE \\ ${ }^{3}$ Specialist Senior Registrar, Neonatal Intensive Care, Pediatric Department, Latifa Hospital, Dubai Health Authority, UAE \\ ${ }^{4}$ Specialist Registrar, Neonatal Intensive Care, Pediatric Department, Latifa Hospital, Dubai Health Authority, UAE
}

Submission: May 3, 2016; Published: July 21, 2016

*Corresponding author: Khaled El-Atawi, Pediatric Department, Latifa Hospital, Dubai, U.A.E,

Email: kelatawi@eim.ae

\begin{abstract}
Intraventricular hemorrhage (IVH) is one of the major complications in premature infants, and its management is quite challenging. This review article describes the pathophysiology, risk factors, and complications of IVH. Further, it explains the current diagnosis and treatment of IVH, along with the recent advances in its management. IVH can occur due to fragility of germinal matrix vasculature, impairment of cerebral auto-regulation, coagulation disorders, genetic factors, and oxygen toxicity. Cranial ultrasonography has been the gold standard for screening in newborns for detecting abnormalities like IVH. However, the need of the hour is diagnostic techniques which not only provide information on brain anatomy, but also related to the hemodynamics. Thus, various techniques are being explored like near infra-red spectroscopy (NIRS), advanced magnetic resonance imaging (MRI) techniques, along with the use of biomarkers. Regarding management of IVH, various invasive and non-invasive methods are used for managing intracranial pressure, respiratory distress, and avoiding complications like bleeding. Nowadays, various bundles of measures are being adopted to decrease the incidence of IVH like delayed cord clamping, minimal handling for first few hours, avoiding head down position, antenatal corticosteroids etc. Establishing standard diagnostic and management practices for IVH can prove to be really helpful in its management and prevention.

Abbreviations: IVH: Intraventricular Hemorrhage; NIRS: Cear Infra-Red Spectroscopy; MRI: Magnetic Resonance Imaging; PHH: PostHemorrhagic Hydrocephalus; PVL: Periventricular Leukomalacia; CBF: Cerebral Blood Flow; BBB: Blood-Brain-Barrier; PDA: Patent Ductus Arteriosus; BP: Blood Pressure; CFOE: Cerebral Fractional Oxygen Extraction; TNF- $\alpha$ :Tumor Necrosis Factor Alpha; CNS: Central Nervous System; ROP: Retinopathy Of Prematurity; BPD: Bronchi pulmonary Dysplasia; RI: Resistive Index; PI: Pulsatility Index; EDBFV: End-Diastolic Blood Flow Velocity; NIRS: Near Infra-Red Spectroscopy; PICU: pediatric intensive care unit; ICH: Cases Of Intracranial Hemorrhage; SWI: SusceptibilityWeighted Imaging; NAA: N-acetyl-aspartate; BOLD: Blood Oxygen Level Dependent; RSNs: Resting State Networks; PHVD: Post-Hemorrhagic Ventricular Dilatation; VP: Ventriculo Peritoneal; VON: Vermont Oxford Network; NICQ: Newborn Improvement Collaborative for Quality
\end{abstract}

\section{Introduction}

Intraventricular hemorrhage (IVH) is one of the major complications in premature infants which continues to pose challenges in neonatal intensive care units (NICUs) worldwide. Globally, 14.9 million babies were born preterm in 2010, and approximately 1.08 million deaths occurred due to preterm birth complications. These preterm infants were noted with increased rates of short-term morbidities like IVH and respiratory distress, and demonstrated poor neurodevelopment outcomes in later years of lives $[1,2]$. For the past five years, several studies conducted worldwide have reported the incidence of IVH between $20 \%-40 \%$ in preterm infants [3-6].
Nevertheless, the incidence of IVH has decreased significantly over the last decade due to worldwide improvements in the neonatal care. A 15-year cohort study revealed that the incidence of IVH has declined from $50.9 \%$ in 1991 to $11.9 \%$ in 2005 . This improvement is primarily attributed to improved practices like use of antenatal corticosteroids, better infrastructure, effective resuscitation skills, appropriate handling of infants, and judicious use of ventilation [7]. However, IVH still poses a major problem as the number of very preterm infants ( $<32$ weeks of gestational age) has increased over the last few years. A study has revealed that the birth rate of very preterm and very low birth weight neonates has increased from $0.87 \%$ in 1996 to $1.10 \%$ in 2008 [8]. Moreover, 
there is a direct correlation between increasing prematurity and severity of IVH [9].

Low psychomotor and mental developmental indices, high incidence of cerebral palsy, and visual impairment are classical triads of preterm infants with IVH. Also, the extent of developmental impairment rises with increasing grade of IVH [10,11]. Post-hemorrhagic hydrocephalus (PHH) and periventricular leukomalacia (PVL) are the two short-term significant sequelae of IVH, while the long-term effects include cognitive, physical, and behavioral abnormalities [12]. These abnormalities create stress on family, educational, health, societal, and financial resources. A progressive increase in hospital cost and length of stay for preterm neonates with IVH and PHH has been noted from the year 2000 to 2010 [13].

IVH continues to be a major complication without any definitive treatment $[9,14,15]$. To address the global burden of IVH, improvements in the diagnostic and management techniques are needed. This review article discusses the various aspects of IVH in preterm infants including the risk factors, pathophysiology, diagnostic tests available, complications (both short and long term), and present trends in the management of this condition. Moreover, the focus of this article is to provide an insight on the advanced diagnostic tools that can be used to improve the screening of IVH, and the bundle of measures that are being adopted for prevention and efficient management.

\section{Getting into the Roots: Risk Factors and Pathophysiology of IVH}

A clear understanding of the pathophysiology is essential for appropriate diagnosis and management of IVH. The pathophysiology of IVH is multifactorial and is primarily ascribed to inherent fragility of the germinal matrix vasculature, disturbance in the cerebral blood flow (CBF), and platelet and coagulation disorders [16].

Fragility of Germinal Matrix Vasculature: The blood-brainbarrier (BBB) is composed of endothelial tight junctions, basal lamina, pericytes, and astrocyte end-feet. Out of all the regions of brain, the germinal matrix exhibits rapid angiogenesis contributing to its high vascular density and cross-sectional area. Due to large number of proliferating, migrating, and maturing neuronal and glial precursor cells, the germinal matrix has high oxygen demand. However, the blood vessels in germinal matrix are immature. Immaturity or weakness of these blood vessels can contribute to fragility of the germinal matrix vasculature. Premature infants bleed into the germinal matrix primarily because of intrinsic weakness in this region [16,17].It has been observed that IVH usually originates from the subependymal veins which are thinwalled and are surrounded by the germinal matrix tissue [18].

Risk factors: Various conditions viz. low gestational age, low birth weight, antenatal maternal hemorrhage, maternal infection/ inflammation, sepsis, hypotension, hypoxia, hypercapnia, seizures, patent ductus arteriosus (PDA), thrombocytopenia, infection, and respiratory distress are major risk factors for IVH. These risk factors are usually responsible for fluctuations in the CBF $[16,17]$. The combination of inherent fragility of germinal matrix and fluctuations in CBF causes the rupture of the vasculature, leading to hemorrhage and thus, filling of blood in the brain's ventricular system $[16,17]$.

Cerebral Autoregulation and Impairment: Cerebral autoregulation constitutes maintenance of constant CBF despite changes in the arterial blood pressure (BP). This is a wellregulated process in adults and children, and acts as a buffering system to maintain a constant tissue perfusion in spite of the fluctuating systemic BP. However, premature infants with IVH exhibit disturbances in this process which leads to pressure passivity of cerebral circulation due to underdevelopment of the process. Therefore, the premature infants are at a high risk of cerebro vascular insult during the transition from the fetal life to the real world $[15,19]$.

Myocardial dysfunction, systemic hypotension, PDA, hypocarbia, and hyperoxia are some of the factors responsible for dysregulation in CBF [20]. Lower left ventricular output, left ventricle stroke volume, cerebral regional oxygen saturation (rSO2), and higher cerebral fractional oxygen extraction (CFOE) have been noted in the infants very soon after birth, followed by an increase in cardiac output just before the occurrence of IVH [21]. In addition, low superior vena cava flow has also been found to be associated with IVH [22]. Ischemia-reperfusion injury has been suggested as a potential cause for the development of IVH $[20,21]$.

Various studies have demonstrated the association of cerebral pressure passivity with the development of the IVH. However, the data remains limited and there is a need to establish the definitive relationship [23-25].

Coagulation Disorders: If there are any platelet or coagulation disorders, hemorrhage may aggravate due to impairment of homeostasis. Thrombocytopenia and disseminated intravascular coagulopathy are considered to be important risk factors for development of IVH $[16,17]$.

Genetic Factors: Mutations in type IV pro collagen gene, factor V Leiden, pro thrombin G20210A, and interleukin (IL)-1 $\beta$ have also been implicated in the development of IVH. Moreover, polymorphisms of inflammatory mediators like IL6 and tumor necrosis factor alpha (TNF- $\alpha$ ) have also been proposed as genetic risk factors for IVH. Thus, it is believed that mutations in genes involved in coagulation, inflammation, and thrombophilia might lead to the occurrence of IVH $[16,17]$.

Oxygen Toxicity: High oxygen levels can be harmful to premature neonates in terms of central nervous system (CNS), pulmonary, and ocular manifestations. This is primarily due to the oxidative stress induced by their rapid environment change from fetal life (poor oxygen supply) to an extra-uterine world (relatively oxygen rich environment). Oxygen derived free radicals are responsible for per oxidation of membrane lipids, inhibition of nucleic acids and protein synthesis, and inactivation of cellular enzymes. Ultimately, oxygen toxicity can lead to conditions like 
IVH, retinopathy of prematurity (ROP), and broncho pulmonary dysplasia(BPD) in preterm babies. [26,27].

Grading: On the basis of intensity and severity, IVH is usually described in 4 grades viz. Grade 1-germinal matrix hemorrhage which is limited to the sub-up endymal parenchyma or minimally involves the ventricle ( $<10 \%$ of the ventricle); Grade 2-intraventricular blood without distension of the ventricular system signifies the spreading of IVH into the ventricle but does not expand or occupy more than $50 \%$ of the ventricle; Grade 3-blood filling and distending the ventricular system and involves more than $50 \%$ of the ventricle; and Grade 4-periventricular venous infarction which is considered as an extensive IVH with parenchymal involvement [12,15]. In a study of 2386 infants, the incidences of IVH Grades 1, 2, 3, and 4 were found to be $25.1 \%$, $7.0 \%, 4.8 \%$, and $5.5 \%$, respectively [5].

Clinical Presentation: IVH can be silent (asymptomatic), saltatory (symptoms may appear one to several hours after birth which includes altered level of consciousness, hypotonia, respiratory distress, and changes in eye movements and position), or catastrophic (evolves over few minutes to hours after birth and signs include seizures, stupor, coma, decerebrate posturing, muscular weakness, and bulging anterior fontanelle) $[15,17]$.

\section{Diagnosis: Present and Future}

As IVH cannot be diagnosed clinically, neuroimaging becomes necessary. As per the Quality Standards Subcommittee of the American Academy of Neurology and the Practice Committee of the Child Neurology Society, routine screening cranial ultrasonography (CS) should be performed in all preterm infants of $<30$ weeks' gestation once between 7-14 days of age and should be optimally repeated between 36 and 40 weeks' postmenstrual age. This helps in the detection of the lesions such as IVH, which influences clinical care, as well as PVL and low-pressure ventriculomegaly providing information about long-term neuro developmental outcomes [12,28].

CS is an inexpensive, non-invasive, and bedside tool which does not cause any major disturbance to the sick infants. However, one major limitation with CS is its limited sensitivity to detect white matter-associated abnormalities like PVL, which are better detected using MRI. As a result, MRI has become increasingly available over the last few years due to its high sensitivity. However, there is insufficient data to prove the utility of MRI in premature infants for the prognosis of the neurodevelopment outcomes [28-31].

Current diagnostic techniques can only look into the anatomy, and not the hemodynamics of the brain. Looking at the future perspective, there is a need for development of advanced techniques which can provide an insight into the brain vascular anatomy along with the hemodynamic information at bedside. Such newer techniques can prove to be beneficial not only as a diagnostic tool, but may also help in the prognosis of the longterm neuro developmental outcomes.
Transcranial Doppler Ultrasonography: Transcranial Doppler ultrasonography is a non-invasive bedside method which can be used to monitor cerebral circulation. It allows direct visualization of the cerebral arteries and easy detection of CBF. Doppler parameters like resistive index (RI), pulsatility index (PI), and end-diastolic blood flow velocity (EDV) have shown a good correlation with the changes in $\mathrm{CBF}$ [32].In premature infants prone to develop IVH, there is a fluctuating pattern in the CBF. Initially, CBF decreases with high RI due to infarction of the blood vessels in the germinal matrix, followed by low RI with bleeding of the vessels [33]. A study conducted in 39 preterm neonates revealed high values of PI and RI and low value of EDV in the patients of IVH. However, most sensitive predictor was found to be EDV [34]. Another study revealed that abnormal RI within the first 72 hours of life was associated with cerebral white matter lesions [33]. Doppler indices have also been seen as important predictors of neonatal morbidities like IVH in fetal growth restriction [35,36].Despite this, the clinical use of transcranial Doppler ultrasonography is limited due to single local measurements of velocities and RI in large arteries. Ultrafast plane-wave Doppler imaging has shown to overcome this limitation, and thus, can become an alternative technique [37].

Near Infra-Red Spectroscopy (NIRS): NIRS is a spectroscopic technique which can be used to measure changes in the oxygenation of the newborn in a non-invasive way. Being a portable, continuous, and non-invasive bedside monitoring technique, it has a great potential for use in NICU. It can be used for measurement of regional $\mathrm{CBF}$ as well as imaging of the brain activity as a function of time. In other words, it allows real-time monitoring of cerebral oxygenation [38,39].

Evidence from the literature has shown that within few days of birth, an abnormal left ventricular output, left ventricular stroke volume, $\mathrm{rSO}_{2}$, and CFOE were observed in IVH infants. Thus, monitoring of cardiac function, $\mathrm{rSO}_{2}$, and CFOE may help in identification of infants at risk for IVH, and come up with considerable preventive actions for the improvement of the developmental outcomes. Since NIRS is used to measure the parameters like $\mathrm{rSO}_{2}$ and $\mathrm{CFOE}$, it can be really helpful in IVH detection and monitoring [21,23,40].

Various studies have shown that NIRS can prove to be a beneficial tool in detection of cerebral autoregulation and its maintenance. A prospective case-control study conducted in a tertiary care pediatric intensive care unit (PICU) correctly identified the cases of intracranial hemorrhage (ICH) in children using NIRS [41]. In another study, NIRS proved to be a very useful tool for monitoring of cerebral oxygenation in preterm neonates on assisted ventilation [42].

A phase II randomized clinical trial was conducted in 166 premature infants to determine if it is possible to stabilize the cerebral oxygenation of extremely preterm infants monitored by cerebral NIRS oximetry. Although the study revealed the stabilization of cerebral oxygenation by the use of NIRS combined 
with a dedicated treatment guideline, larger randomized studies are needed to assess the long-term benefits and harms of NIRS [43]. Currently, an observational study "Cerebral Oxygenation and Autoregulation in Preterm Infants (Early NIRS)" is ongoing to identify the preterm infants at highest risk for brain injury or death using NIRS [44]. Once a solid evidence is built, NIRS can attract a large market.

Advanced MRI Techniques: Nowadays, advanced MRI techniques in focus include MR spectroscopy (MRS), functional MRI (f MRI), and susceptibility-weighted imaging (SWI) for evaluation of premature infants. In contrast to MRI, ${ }^{1} \mathrm{H}-\mathrm{MRS}$ measures the signal of protons attached to carbohydrates, fatty acids, and lipids involved in the brain biochemical process and examines the metabolites like $\mathrm{N}$-acetyl-aspartame (NAA), creatine, choline (Cho), and lactate in the preterm brain. It has been suggested to be a useful tool in measurement of the neuro developmental outcomes. However, the utility of MRS is not well established till now. The method is not absolutely quantified and measures general outcomes like overall development quotient [31]. On the other hand, f MRI is a functional neuroimaging technique that measures the neural activity by assessing spontaneous, low-frequency fluctuations in blood oxygen level dependent (BOLD) signal. These fluctuations are consistent within the different parts of the brain that together constitute the resting state networks (RSNs). The spatial topography of these RSNs is repeated in response to various tasks like cognitive, motor, and sensory. Recent studies have shown the utility of $f$ MRI in neuro developmental studies as well as impact of brain abnormalities on RSN development [31,45]. SWI is another advanced MRI technique that is gaining popularity in pediatric neuroimaging. It helps in easy visualization of paramagnetic substances like de oxy hemoglobin, intracellular methemoglobin, and hemosiderin. It has been found to have greater sensitivity than conventional MRI in detection of small hemorrhages [46]. However, further studies are needed to establish the role of MRS, f MRI, or SWI in detection of IVH as well as prediction of long-term neurological outcomes.

Biomarkers: Recently, there is a growing interest on the use of biomarkers for early diagnosis of IVH. Activin A, a growth factor belonging to the transforming growth factor-beta super family, plays an important role in the physiologic response to any brain injury. Preterm neonates with IVH have been noticed with increased activin levels, and thus, it seems to be a promising biomarker for IVH. Another biomarker, S100b is synthesized by astrocytes and is reported to be a predictor of IVH and neonatal mortality. IL-6 and erythropoietin have also been shown to be potential biomarkers for IVH $[47,48]$.

The way ahead: Although newer diagnostic techniques and biomarkers appear to be promising in diagnosis of IVH, there is a need for larger number of studies to prove their benefits. Once stronger evidence is built, these techniques and biomarkers can prove to be very helpful in early diagnosis and prediction of IVH, and thus, can also help in prevention of long-term complications.

\section{Management Of IVH In Preterm Infants: Present and Future}

IVH and Associated Complications: The occurrence of IVH is associated with both short-term and long-term complications. IVH can prove to be fatal in many premature infants. Those who survive, may experience post-hemorrhagic ventricular dilatation (PHVD) and PHH. Ultimately, PHH is associated with various degrees of neurodevelopment disabilities.IVH may also lead to white matter abnormalities like PVL, and thus, causing abnormalities like cognitive impairment, decreased visual fields, and spastic diplegia [12].

In the first few days of life, infants with IVH are at high risk of seizures, PHH, and PVL, while they may experience neuro developmental disabilities at later stages. They may develop cerebral palsy, mental retardation, or cognitive dysfunctions. While the premature infants with Grade3-4 IVH are at high risk of cerebral palsy and mental retardation, infants with Grade1-2 IVH are also at risk of developmental disability. A large number of preterm infants with Grade 3-4 IVH suffer from cognitive handicaps, and need special education, thus, it becomes important to have appropriate measures for the prevention and management of IVH [12].

Management: Although there is no definitive treatment for IVH, conventional strategy is managing BP, intracranial pressure, and respiratory stress of neonates; avoiding complications like re-bleeding; and correction of coagulation disorders which might influence the progression of IVH. In addition, management of IVH aims at screening for IVH-associated sequelae like PHH and PVL, and their treatment. In the last few years, various invasive and non-invasive methods have been put forward to prevent as well as manage the IVH and the development of PHH $[9,12,14]$.

Invasive Methods and Implications for Practice: Ventricular peritoneal (VP) shunting has been the best definitive treatment for PHH. It leads to permanent CSF diversion in infants with PHH. However, the method is associated with poor longterm outcomes, shunt infections, and risk of shunt obstructions leading to shunt failure. This has led to the emergence of the use of temporary shunt placements which can postpone the insertion of VP shunts. Delayed insertion allows the infants to be mature and well-nourished, and thus, reduces the risks associated with the permanent shunt insertion. The two major methods of temporary CSF diversion are ventricular reservoirs and ventriculo- subgaleal (VSG) shunts [9,15].

Subcutaneous reservoir is another frequently used method for the management of PHH. There is no need of repeated needle tracks with subcutaneous reservoirs, and thus, avoids brain injury. Early lumbar punctures (LP) or ventricular taps have also been considered as a way to avoid PHH and protect the brain from excessive pressure. These methods are often adequate to treat the transient phase of $\mathrm{PHH}$, and have shown to decrease the need for surgical intervention or shunt insertion $[9,15]$.

However, none of the above mentioned treatment can be recommended as a definitive treatment. Ventricular reservoirs 
and VSG shunts are associated with infections, and their effect on neurodevelopment outcome is unknown at present. Also, there is a lack of evidence that early LP/ventricular taps reduce the risk of shunt dependency, death or poor neurodevelopment outcomes. Moreover, repeated taps as well as subcutaneous reservoirs have been associated with infections like meningitis and ventriculitis; and the removal of CSF with these procedures is intermittent $[9,15]$.

Non-Invasive Methods and Implications for Practice: Antenatal corticosteroids have shown to increase the survival rate and reduce the rate of IVH in premature children. The combination of antenatal corticosteroids and Vitamin $\mathrm{K}$ has also shown to decrease the incidence of IVH in infants $[9,49]$. Besides this, antenatal indo mechanic also seems to be promising. A review of 19 trials with 2872 infants revealed that prophylactic treatment with indo mechanic can reduce the incidence of PDA as well as severe IVH. However, there was no evidence that suggested the effect of indo mechanic on long-term neurodevelopment outcomes [50]. Recently, a risk prediction model was developed to analyze the effect of prophylactic indo methacin in very low birth weight infants. The study revealed that prophylactic indo methacin was associated with a lower risk-adjusted incidence of severe IVH [51]. However, another study revealed that prophylactic indo methacin administered before 6 hours of life is not associated with lower incidence of IVH [52]. Evidence from the literature also suggests that factor VII may be an effective agent in prevention of bleeding, and thus, can be a potential treatment for IVH $[12,53]$.

Although these pharmacological interventions have shown a good potential, there is limited data regarding their effect on long-term neurodevelopment outcomes, and further studies are needed. Looking at the positive side, these latest trends from literature suggest that further research can be focused on antenatal care for treating IVH and its associated complications.

Drugs which are not recommended anymore: Recently, a review of 12 controlled trials with 982 infants was conducted to determine the effect of postnatal administration of phenol barbital on the risk of IVH, neuro developmental impairment or death. The review revealed that there is lack of evidence that postnatal phenol barbital is effective in preventing IVH. Furthermore, it is associated with an increased need for mechanical ventilation, and thus, phenol barbital cannot be recommended as prophylaxis to prevent IVH in preterm infants [54]. Earlier, ethamsylate was also suggested to have a role in reducing IVH in preterm infants. However, a review of 7 trials with 1410 preterm infants revealed that although IVH was reduced in infants, there was no improvement in developmental outcomes, and thus, its use is not recommended for IVH [55].

Introduction of bundle of measures: Vermont Oxford Network (VON) was developed in 1980s to change the landscape of neonatal care, and is comprised of healthcare professionals representing NICUs around the whole world. It works via Newborn Improvement Collaborative for Quality (NICQ) to continually improve the quality, safety, and value of newborn care. Under NICQ 2000, five NICUs formed a group with a common goal of decreasing the incidence of IVH and PVL among neonates $\leq 1500 \mathrm{~g}$. The evidence-based quality improvement approach suggests that some potentially better practices were developed and implemented. The practices included optimal peripartum management, such as resuscitation, maintaining the body's temperature at $\geq 36^{\circ} \mathrm{C}$, optimal surfactant delivery; early neonatal management; measures to minimize pain and stress via developmental care and minimal handling; maintenance of neutral head position; fluid volume therapy for hypotension; indo methacin prophylaxis in first 24 hours of birth; appropriate ventilation; avoidance of routine suctioning; and limiting the use of sodium bicarbonate and postnatal dexamethasone [56,57].

Since then, various NICUs have been introducing certain bundle of measures to decrease the incidence of IVH. These measures are concerned with the steps taken during delivery, immediate care after delivery, and infants' first few days of birth. Bed well et al. [58] introduced an IVH prevention bundle based on VON NICQ 2000 and evidence-based research on cerebral circulation in OU Medical Center, Oklahoma (US).

The bundle of measures comprised of avoiding head down position, keeping head of bed at 15 degrees, midline positioning of head for $72 \mathrm{~h}$, avoiding rapid flushes, and maintaining body's temperature $>36.5^{\circ} \mathrm{C}$. This led to significant decrease in the incidence of severe IVH [58]. A Swedish Medical Center NICU also worked to create a standardized care bundle which included educating the clinical staff on IVH reduction program, giving antenatal corticosteroids and magnesium to mothers for neuroprotection, delayed cord clamping and prevention of hypothermia in delivery room, midline head positioning for first $72 \mathrm{~h}$, minimal handling and stimulation, and slow infusions of fluids, if needed. Though this medical center didn't demonstrate significant reduction in IVH, it is currently aiming to improve its bundle of measures [59]. However, another NICU in Philadelphia showed measurably reduced incidence of IVH $(5.1 \%$ from $8.3 \%$ ) after introduction of bundle of measures like midline head positioning, minimal handling, increasing IVH awareness, and standardizing infusion rates for boluses/blood product [60]. A working group in Germany also revealed the decrease of incidence of IVH from $22.1 \%$ to $10.5 \%$ after the introduction of bundle of preventive measures; the major measures adopted were: preference of caesarean section as a method of delivery, delayed clamping of the umbilical cord, and additional dose of beta methadone during pregnancy [4]. A literature review also supported the technique of delayed cord clamping in decreasing the risk of IVH and improving neonatal morbidity [61].

The way ahead: On the basis of available evidences, there is a need to direct our focus on proper management of IVH with adequate measures for its prevention. Appropriate maternal care and method of delivery might help in prevention of IVH. After birth, a new-born should be under intensive care for first few days of life and be monitored for oxygen saturation levels, and 
development of any complications like PHH, PVL, ROP. Looking at the future perspective, research can be carried out in the fields of robotics, stem cells, and in utero therapies. Interdisciplinary approaches can be adopted that can combine the beneficial effects of antenatal care, pediatric intensive care, as well as neonatal medicines for IVH.

\section{Conclusion}

In conclusion, IVH continues to be a major complication in premature infants. It may lead to development of PHH and PVL in the new-born period, and cause cognitive, physical, and behavioral abnormalities in the long-term period. The major concern regarding IVH is lack of definite diagnostic and management techniques. CS remains to be a method of choice for detection of brain abnormalities like IVH. Although better techniques like Doppler ultrasonography and NIRS seems to be promising, their use is limited and solid evidences need to be established. Furthermore, advanced MRI techniques and biomarkers can also help in the better diagnosis of IVH. Besides the advanced diagnostic tools, standard treatment and prevention guidelines need to be established for IVH.

Currently, there is no definitive treatment for IVH, and the invasive methods pose a great risk for infections. Moreover, there is limited data regarding the effect of invasive and non-invasive methods on long-term neurodevelopment outcomes. Recently, a bundle of measures has been adopted by various NICUs like caesarean delivery, delayed cord clamping, minimal handling of infants, avoiding head down position, midline positioning of head for $72 \mathrm{~h}$, keeping head of bed up at 15 - 20 degrees, slow infusion of fluids, and giving antenatal corticosteroids for IVH prevention. Once the standard treatment is established, it will be very helpful in controlling the IVH and its-associated mortality and long-term neurodevelopment outcomes.

\section{References}

1. Blencowe H, Cousens S, Chou D, Oestergaard M, Say L, et al, (2013) Born Too Soon Preterm Birth Action Group. Born too soon: the global epidemiology of 15 million preterm births. Reprod Health 10(1): 10 Suppl 1: S2.

2. Gladstone M, Oliver C, Van den Broek N (2015) Survival, morbidity, growth and developmental delay for babies born preterm in low and middle income countries - a systematic review of outcomes measured. PLoS One 10(3): e0120566.

3. Mulindwa MJ, Sinyangwe S, Chomba E (2014) The Prevalence of Intraventricular Haemorrhage and Associated Risk Factors in Preterm Neonates in the Neonatal Intensive Care Unit at the University Teaching Hospital, Lusaka, Zambia. Med J Zambia 39(1): 16-21.

4. Schmid MB, Reister F, Mayer B, Hopfner RJ, Fuchs H (2013) Prospective risk factor monitoring reduces intracranial hemorrhage rates in preterm infants. Dtsch Arztebl Int 110(29-30): 489-496.

5. Ahn SY, Shim SY, Sung IK (2015) Intraventricular Hemorrhage and Post Hemorrhagic Hydrocephalus among Very-Low-Birth-Weight Infants in Korea. J Korean Med Sci 30(1): 52-58.

6. Adegoke SA, Olugbemiga AO, Bankole KP, Tinuade OA (2014) Intraventricular hemorrhage in newborns weighing $<1500$ g:Epidemiology and short-term clinical outcome in a resource-poor setting. Ann Trop Med Public Health 7(1): 48-54.

7. Marba ST, Caldas JP, Vinagre LE, Pessoto MA (2011) Incidence of periventricular/intraventricular hemorrhage in very low birth weight infants: a 15-year cohort study. J Pediatr (Rio J) 87(6): 505-511.

8. Rüegger C, Hegglin M, Adams M, Bucher HU (2012) Population based trends in mortality, morbidity and treatment for very preterm- and very low birth weight infants over 12 years. BMC Pediatr 12: 17.

9. Shooman D, Portess H, Sparrow O (2009) A review of the current treatment methods for posthaemorrhagic hydrocephalus of infants. Cerebrospinal Fluid Res 6: 1.

10. Klebermass-Schrehof K, Czaba C, Olischar M, Fuiko R, Waldhoer T, et al. (2012) Impact of low-grade intraventricular hemorrhage on longterm neuro developmental outcome in preterm infants. Childs Nerv Syst 28(12): 2085-2092.

11. Bolisetty S, Dhawan A, Abdel-Latif M, Bajuk B, Stack J (2014) New South Wales and Australian Capital Territory Neonatal Intensive Care Units' Data Collection. Intraventricular hemorrhage and neuro developmental outcomes in extreme preterm infants. Pediatrics 133(1): 55-62.

12. McCrea HJ, Ment LR (2008) The diagnosis, management, and postnatal prevention of intraventricular hemorrhage in the preterm neonate. Clin Perinatol 35(4): 777-792.

13. Christian EA, Jin DL, Attenello F, Wen T, Cen S, et al. (2015)Trends in hospitalization of preterm infants with intraventricular hemorrhage and hydrocephalus in the United States, (2000-2010). Fluids and Barriers CNS. 17(1):260-269.

14. Hinson HE, Hanley DF, Ziai WC (2010) Management of intraventricular hemorrhage. Curr Neurol Neurosci Rep 10(2): 73-82.

15. Robinson S (2012) Neonatal posthe morrhagic hydrocephalus from prematurity: pathophysiology and current treatment concepts. J Neuro surge Pediatr 9(3): 242-258.

16. Ballabh P (2014) Pathogenesis and prevention of intraventricular hemorrhage. Clin Perinatol 41(1): 47-67.

17. Ballabh $P$ (2010) Intraventricular hemorrhage in premature infants: mechanism of disease. Pediatr Res 67(1): 1-8.

18. Anstrom JA, Brown WR, Moody DM, Thore CR, Challa VR (2004) Subependymal veins in premature neonates: Implications for hemorrhage. Pediatr Neurol 30(1): 46-53.

19. du Plessis AJ (2009) The role of systemic hemodynamic disturbances in prematurity-related brain injury. J Child Neurol 24(9): 1127-1140.

20. Noori S, Seri I (2015) Hemodynamic antecedents of peri/ intraventricular haemorrhage in the very preterm neonates. Semin Fetal Neonatal Med 20(4): 232-237.

21. Noori S, McCoy M, Anderson MP, Ramji F, Seri I (2014) Changes in the cardiac function and cerebral blood flow in relation to peri/ intraventricular haemorrhage in extremely preterm infants. J Pediatr 164(2): 264-270.

22. Kluckow M, Evans N (2000) Low superior vena cava flow and intraventricular hemorrhage in preterm infants. Archs Dis Child Fetal Neonatal Ed 82(3): F188-F194.

23. Verhagen EA, Ter Horst HJ, Keating P, Martijn A, Van Braeckel KN (2010) Cerebral oxygenation in preterm infants with germinal matrixintraventricular hemorrhages. Stroke 41(12): 2901-2907.

24. O'Leary H, Gregas MC, Limperopoulos C, Zaretskaya I, Bassan H, et al. (2009) Elevated cerebral pressure passivity is associated with prematurity-related intracranial hemorrhage. Pediatrics 124(1): 302309.

25. Soul JS, Hammer PE, Tsuji M, Saul JP, Bassan H, et al. (2007) Fluctuating pressure-passivity is common in the cerebral circulation of sick premature infants. Pediatr Res 61(4): 467-473.

26. Perrone S, Tataranno ML, Stazzoni G, Buonocore G (2012) Oxidative stress and free radicals related diseases of the newborn. Adv Biosci Biotechnol 3: 1043-1050.

27. Patel DN, Goel A, Agarwal SB, Garg P, Lakhani KK (2003) Oxygen toxicity. J Indian Acad Clin Med 4(3): 234-237. 
28. Ment LR, Bada HS, Barnes P, Grant PE, Hirtz D, et al. (2002) Practice parameter: neuroimaging of the neonate: report of the Quality Standards Subcommittee of the American Academy of Neurology and the Practice Committee of the Child Neurology Society. Neurology 58(12): 1726-1738.

29. Rademaker KJ, Uiterwaal CS, Beek FJ, van Haastert IC, Lieftink AF, et al. (2005) Neonatal cranial ultrasound versus MRI and neuro developmental outcome at school age in children born preterm. Arch Dis Child Fetal Neonatal Ed 90(6): F489-F493.

30. Leijser LM, Cowan FM (2007) 'State-of-the-Art' Neonatal Cranial Ultrasound. Ultrasound 15(1): 6-17.

31. Panigrahy A, Wisnowski JL, Furtado A, Lepore N, Paquette L, et al (2012) Neuroimaging biomarkers of preterm brain injury: toward developing the preterm connectome. Pediatr Radiol 42(1): S33-61.

32. Naqvi J, Yap KH, Ahmad G, Ghosh J (2013) Transcranial Doppler ultrasound: a review of the physical principles and major applications in critical care. Int J Vasc Med 629378.

33. Argollo N, Lessa I, Ribeiro S (2006) Cranial Doppler resistance index measurement in preterm newborns with cerebral white matter lesion. J Pediatr (Rio J) 82(3): 221-226.

34. Elhameed AMA, Fouda EM (2011) Transcranial ultrasound Doppler parameters in cerebral vessels may predict intracerebral hemorrhage in preterm newborns. Egyptian Journal of Radiology and Nuclear Medicine 42(1): 69-75.

35. Marsoosi V, Bahadori F, Esfahani F, Ghasemi-Rad M (2012) The role of Doppler indices in predicting intra ventricular hemorrhage and perinatal mortality in fetal growth restriction. Med Ultrason 14(2): 125-132.

36. Mahale N, Khanal B, Mahale A, Fernandes M, Rao P, et al. (2015) Doppler prediction of adverse perinatal outcome in intrauterine growth restriction. Int J Reprod Contraception Obstet Gynecol 4(1): 119-130.

37. Demené C, Pernot M, Biran V, Alison M, Fink M, et al. (2014) Ultrafast Doppler reveals the mapping of cerebral vascular resistivity in neonates. J Cereb Blood Flow Metab 34(6): 1009-1017.

38. Ioniță N, Dima MA, Ilie C, Agoston-Vas AE, Nyiredi A (2013) Nearinfrared spectroscopy in the neonatal intensive care unit-Aliterature review. Jurnalul Pediatrului 16(64): 70-73.

39. Murkin JM, Arango M (2009) Near-infrared spectroscopy as an index of brain and tissue oxygenation. Br J Anaesth 103(1): i3-13.

40. Alderliesten T, Lemmers PM, Smarius JJ, van de Vosse RE, Baerts W, (2013) Cerebral oxygenation, extraction and autoregulation in very preterm infants who develop peri-intraventricular hemorrhage. J Pediatr 162(4): 698-704.

41. Salonia R, Bell MJ, Kochanek PM, Berger RP (2012) The utility of near infrared spectroscopy in detecting intracranial hemorrhage in children. J Neurotrauma 29(6): 1047-1053.

42. Ioniță N, Dima MA, Agoșton-Vas AE, Ilie C (2014) Monitoring of cerebral oxygenation using near infrared spectroscopy in preterm neonates on assisted ventilation. Jurnalul Pediatrului 17 (65-66): 50-54.

43. Hyttel-Sorensen S, Pellicer A, Alderliesten T, Austin T, van Bel F, et al. (2015) Cerebral near infrared spectroscopy oximetry in extremely preterm infants: phase II randomised clinical trial. BMJ 350:g7635.

44. Ongoing study: Cerebral Oxygenation and Autoregulation in Preterm Infants (Early NIRS) (2015).
45. Smyser CD, Snyder AZ, Shimony JS, Blazey TM, Inder TE, (2013) Effects of white matter injury on resting state f MRI measures in prematurely born infants. PLOS One 8(7): e68098.

46. Intrapiromkul J, Northington F, Huisman TA, Izbudak I, Meoded A, et al. (2013) Accuracy of head ultrasound for the detection of intracranial hemorrhage in preterm neonates: comparison with brain MRI and susceptibility-weighted imaging. J Neuroradiol 40(2): 81-8.

47. Andrikopoulou M, Almalki A, Farzin A, Cordeiro CN, Johnston MV, et al. (2014) Perinatal biomarkers in prematurity: early identification of neurologic injury. Int J Dev Neurosci 36: 25-31.

48. Douglas-Escobar M, Weiss MD (2013) Biomarkers of brain injury in the premature infant. Front Neurol 3: 185.

49. Wong D, Abdel-Latif M, Kent A (2014) Antenatal steroid exposure and outcomes of very premature infants: a regional cohort study. Arch Dis Child Fetal Neonatal Ed 99(1): F12-20.

50. Fowlie PW, Davis PG, McGuire W (2010) Prophylactic intravenous indo mechanic for preventing mortality and morbidity in preterm infants. Cochrane Database Syst Rev (7): CD000174.

51. Luque MJ, Tapia JL, Villarroel L, Marshall G, Musante G, et al; (2014) Neocosur Neonatal Network. A risk prediction model for severe intraventricular hemorrhage in very low birth weight infants and the effect of prophylactic indo methacin. J Perinatol 34(1): 43-48.

52. Mirza H, Oh W, Laptook A, Vohr B, Tucker R, Stonestreet BS (2013) Indomethacin prophylaxis to prevent intraventricular hemorrhage: association between incidence and timing of drug administration. J Pediatr 163(3): 706-710.

53. Veldman A, Josef J, Fischer D, Volk WR (2006) A prospective pilot study of prophylactic treatment of preterm neonates with recombinant activated factor VII during the first 72 hours of life. Pediatr Crit Care Med 7(1): 34-39.

54. Smit E, Odd D, Whitelaw A (2013) Post natal phenol barbital for the prevention of intraventricular haemorrhage in preterm infants. Cochrane Database Syst Rev. 13(8): CD001691.

55. Hunt R, Hey E (2010) Ethamsylate for the prevention of morbidity and mortality in preterm or very low birth weight infants. Cochrane Database Syst Rev 20(1): CD004343.

56. McLendon D, Check J, Carteaux P, Michael L, Moehring J, et al. (2003) Implementation of potentially better practices for the prevention of brain hemorrhage and ischemic brain injury in very low birth weight infants. Pediatrics 111(4 Pt 2): e497-503.

57. Whitfield J, Charsha D, Sprague P (2001)In search of excellence--the Neonatal Intensive Care Quality Improvement Collaborative. Proc (Bayl Univ Med Cent) 14(1): 94-97.

58. Bedwell SM, Bright B, Sekar KS. (2015) Decrease in the incidence of Intraventricular hemorrhage (IVH) after the introduction of an IVH prevention bundle in the NICU.

59. Nervik T, Moore L, Ryan A, Nielson S, Watkins K, et al. (2015) Reducing Intraventricular Hemorrhage Using a Care Bundle.

60. Christ L, Barber J, Murray A, Dunleavy M, Stoller J, et al. (2015) Reducing intraventricular hemorrhage in a level III neonatal intensive care unit. BMJ Qual Saf 24(11): 731-732.

61. Brocato B, Holliday N, Whitehurst RM, Lewis D, Varner S (2016) Delayed Cord Clamping in Preterm Neonates: A Review of Benefits and Risks. Obstet Gynecol Surv 71(1): 39-42. 